\title{
Entre el Paternalismo Conocido y la Participación por Conocer Reflexiones junto con sectores del barrio Buceo a partir de una intervención comunitaria
}

\section{Beetween Wellknown Paternalism and to-be-Known Participation Thinking about a community intervention with people from Buceo neighborhood}

\author{
Lic. Clara Netto * \\ Ps. Alicia Rodríguez* \\ Lic. Susana Rudolf*
}

Resumen

Se parte de una intervención comunitaria que desarrolla un equipo docente del curso "Técnicas de Atención Comunitaria" del Area de Salud de la Facultad de Psicología de la Universidad de la República en Montevideo, Uruguay.

La respuesta a un pedido por parte de integrantes de una Capilla del barrio Buceo posibilita el desarrollo de una estrategia de trabajo que tiene como sustento la desideologización y la concientización en relación a diversos aspectos de la ideología dominante que se ponen en juego en la relación que los agentes comunitarios establecen con los sujetos de sus prácticas sociales.

Desde allí se reflexiona sobre el sentido del cambio social que se persigue, tanto por parte de dichos agentes como de los técnicos que intervienen desde el marco de una Psicología Comunitaria considerada en su carácter esencialmente político.

Un análisis de la coyuntura social permite otorga fundamentos a la intervención desarrollada a la vez que analizar la implicación de los profesionales en el proceso dialógico que supone esta perspectiva de intervención comunitaria.

Palabras Claves: Intervención comunitaria. Cambio social.

\section{Abstract}

The paper analyses a community intervention developed by professors who belong to the course Techniques in Community Service, Health Department, School of Psychology, University of the Republic, Montevideo, Uruguay.

The response to a demand put by members of a chapel allows the development of a strategy based on gaining awareness about some aspects of dominant ideology affecting the relationship of community agents with recipients of their social practices.

\footnotetext{
* Facultad de Psicología, Universidad de la República. Montevideo, Uruguay.

e-mail: cnetto@psico.edu.uy - e-mail: aliciar@psico.edu.uy
} 
Reflection goes to the kind of social change aimed at by community psychology if considered from a political point of view. The analysis of social situation gives ground to intervention and allows to think about professionals implication in the dialogic process here displayed.

\section{Características Generales de la Experiencia}

\section{1- Inicio del trabajo y marco institucional en el que se desarrolla la intervención}

En mayo de 1997, el diácono de la Capilla Santa Elena acude a la Facultad de Psicología para pedir asesoramiento técnico y apoyo a las actividades sociales que la Pastoral Social viene realizando con sectores de pobreza extrema del barrio Buceo de Montevideo, en el que también se encuentra la Facultad de Psicología ${ }^{2}$.

Uno de los pedidos se centra en un apoyo metodológico que permita modificar las actitudes paternalistas y sobreprotectoras a las que son proclives los voluntarios que trabajan en la Pastoral Social, e incrementar el involucramiento en las distintas iniciativas de la población con la que trabajan.

El marco institucional desde el que se formula el pedido es la Iglesia Católica. La forma de acción de la Capilla hacia la comunidad en la que está inserta pasa por lo evangélico y también por lo promocional. Tal como sus integrantes afirman, a partir del Concilio Vaticano II hay una intencionalidad de dejar de lado las prácticas asistencialistas sustituyéndolas por la de promoción social.

La Teología de la Liberación ha sido una contribución decisiva de América Latina en las prácticas de la iglesia. Existen serias tensiones ideológicas entre conservadores y progresistas. La marca del asistencialismo sin embargo, es muy difícil de borrar, de allí la preocupación por otro enfoque en la prácti- ca social que llevó al diácono a conectarse con la Facultad de Psicología, contando para ello con la aprobación de los integrantes de la Pastoral Social.

Los miembros de la Pastoral Social tienen trayectorias de militancia social y política y muchas de las propuestas que llevan adelante son cuestionadas por otros miembros de la iglesia. Vienen desarrollando trabajos con familias de extrema pobreza de la zona: un almacén comunitario, un espacio recreativo con niños y talleres para adultos. Apoyan actividades solidarias (Banco de Medicamentos), impulsan encuentros y debates de carácter social, por ejemplo, charlas sobre el Movimiento de los Sin Tierra de Brasil.

A su vez, la Capilla Santa Elena forma parte de una experiencia inédita de participación barrial que involucra a varios actores sociales de la zona : el sindicato del vidrio, la directora de una de las escuela, integrantes de clubes deportivos, iglesias Católica y Metodista, Club de Leones, Asociación de Jubilados y Concejales de tres Centros Comunales Zonales de la Intendencia $\mathrm{Mu}$ nicipal de Montevideo. Se nuclean desde hace dos años en el Encuentro Barrial del Buceo que se reúne mensualmente para realizar acciones tendiente al mejoramiento de la calidad de vida de los habitantes de la zona.

Las matrices de participación social del Uruguay están fuertemente ligadas a los modelos gremiales y sindicales.

La participación territorial no tuvo un desarrollo importante a excepción del fuerte movimiento cooperativo de viviendas por ayuda mutua. Sin embargo, al ganar el Fren- 
te Amplio (conjunción de movimientos y partidos de izquierda) la Intendencia Municipal de Montevideo en 1990, impulsó la participación ciudadana en la gestión local a partir de una propuesta de descentralización.

Analizando la experiencia del Encuentro Barrial vemos que logra trascender los aspectos de mera demanda y se coloca en un lugar de negociación con el Estado y de formulación de propuestas, que le permite pensar en las problemáticas sociales de la zona con la riqueza de la diversidad de actores sociales que lo componen.

\section{2- Características de la zona}

Ante de pasar al desarrollo de la intervención, unas palabras sobre el barrio Buceo ${ }^{3}$.

Buceo presenta características heterogéneas en cuanto a población y construcciones. Es una zona costera, aledaña a una amplia playa, con edificaciones bajas, de casas con pequeños jardines en calles arboladas, con conjuntos habitacionales que agrupan diferente tipo de población. Cuenta con un adecuado servicio de locomoción y comercio en general. La construcción de un shopping hace diez años modifició el espacio geográfico y vincular.

Sus habitantes están ubicados entre los sectores medios de la población, percibiéndose una tendencia a ser ocupada por sectores de mayor poder adquisitivo. Sin embargo, los viejos pobladores, fundamentalmente italianos y españoles inmigrantes y sus descendientes directos, tienden a quedarse en las casas que construyeron.

Muchas de esas viviendas están ocupadas por varias familias o no pueden ser mantenidas, convirtiéndose en fincas ruinosas. Hay importantes bolsones de pobreza que tienden a no verse tras las fachadas de las casas. La mendicidad infantil y los niños la- vando parabrisas de lo autos en los semáforos junto a problemas de delincuencia, drogas y violencia en general, son denunciados por los vecinos como una de las características nuevas de una zona antes tranquila.

Los habitantes de raza negra que viven en esta zona, le dan al barrio la impronta de expresiones culturales propias del carnaval uruguayo. El toque de tambores ("llamadas") es una fiesta que feriado y fines de semana congrega a numerosísimos jóvenes del barrio.

Un club de fútbol, el Huracán Buceo, contribuye al sentimiento de pertenencia a la zona. Numerosos clubes de baby fútbol y basquetbol además de la playa en verano, son las formas de esparcimiento de sus habitantes.

Las fuente de ocupación propia del barrio no son muchas. No tenemos datos exactos pero la mayoría de sus habitantes debe desplazarse a otros barrios para trabajar. Además de los comercios y los puestos de pesca de la rambla, la zona cuenta con una fábrica de vidrio con un sindicato bastante activo y vinculado al barrio. En el mes de abril de 1999 la patronal decidió el cierre de la plantea de producción, y desde ese momento los trabajadores comenzaron una prolongada ocupación con el apoyo masivo de los vecinos.

\section{3-Intervención desarrollada}

A partir del pedido de intervención, nos planteamos una primera etapa de familiarización realizando entrevistas y acercándonos a los distintos espacios y grupos conformados. Luego formulamos una estrategia que buscó profundizar en el diagnóstico y en la construcción de propuestas de acción en los distintos ámbitos de trabajo.

Nos centraremos aquí en la intervención realizada con los integrantes de la Pastoral Social. 
$\mathrm{El}$ aspecto central que surge en esta primera etapa es el relacionamiento entre los voluntarios de la Pastoral que e hacen cargo de desarrollar las actividades y las familias destinatarias. Los problemas de comunicación, la puesta en juego de expectativas mutuas no siempre coincidentes, las dificultades para cumplir con los objetivos que se plantean, imponen una reflexión sobre su accionar. Esto se realizó a partir de cuatro talleres consecutivos.

En un primer momento buscamos conocer y compartir sus experiencias anteriores de participación guiados por las preguntas "¿Quiénes somos, de dónde venimos y hacia dónde vamos?". A partir de este recorrido se pudieron contactar con sus propias motivaciones y necesidades que los llevan a participar socialmente. Surge la pregunta : "¿Quiénes son los necesitados?".

En un segundo momento proponemos la realización de una matriz que ayude a analizar las prácticas que realizan incluyendo los siguientes ítems : ¿Qué hacen, quiénes lo hacen, para qué, con quiénes, cómo y desde dónde (marco teórico referencial)?

Se problematizó la visión -predominantemente negativa- que los integrantes de la Pastoral tiene de la población con la que trabajan y el tipo de vínculo que establecen. Surge la pregunta : " $¿$ Trabajar con o trabajar para la gente?".

Plantean el deseo de trabajar con ellos pero a la vez la imposibilidad por las características que les asignan. En la matriz que construyen se evidencian las contradicciones entre una intención liberadora y de ruptura con una práctica asistencialista y la visión de los otros como seres de carencias y con atributos negativos ("vienen a recibir y no a dar, son cómodos, no les gusta trabajar, no tienen esperanzas, no entienden qué queremos de ellos, manipulan, mienten"). Ver esto por escrito les produjo un fuerte impacto. Uno de los integrantes expresó : "Se me vino el mundo abajo".
También se trabajó sobre algunas características de la dinámica grupal que dificultaban la integración de otras personas a pesar de la queja permanente por la sobrecarga de tareas.

\section{Problemas, Tensiones e Interrogantes que Surgen de la Experiencia.}

\subsection{Análisis del pedido}

A partir de las distintas actividades pudimos observar y empezar a trabajar la tensión existente entre una intención educativa y promocional, y una práctica asistencialista: los integrantes de Pastoral Social rechazan la relación paternalista que establecen con la gente, pero a la vez, no encuentran la forma para sustraerse de ella.

Parten de referentes conceptuales e ideológicos tales como la Teología de la Liberación y la Educación Popular, citando materiales de Paulo Freire y de Franz Hinkelammert. Sin embargo, la práctica que llevan a cabo está marcada por una percepción de los excluidos "concretos" como seres apáticos, cómodos, manipuladores, poco dispuestos a colaborar en los objetivos que ellos se plantean. De esta manera, se atribuye a las personas sobre las que se pretende operar cambios, toda la dificultad y resistencia para generarlos.

Sabemos que este fenómeno no es exclusivo de estos agentes sociales, ya que lo podemos relacionar con el llamado "síndrome fatalista", sobre el que Ignacio Martín Baró (1987) ha reflexionado a propósito de la percepción y autopercepción de los latinoamericanos y de las dificultades que esto acarrea en el trabajo comunitario.

Notamos en los integrantes de la Pastoral Social, una importante dificultad para comprender las necesidades, los valores, las 
formas de vida de la población con la que trabajan, que son diferentes a las de ellos mismos. En la misión que se les tiene asignada a los pobres tanto desde algunos sectores de la iglesia como desde núcleos de la izquierda más tradicionales, se oscila entre un lugar protagónico en la generación de los cambios sociales, y la atribución de un lugar pasivo y de indefensión, o de franco papel reaccionario.

Gloria Perdomo (1988) plantea cuatro perfiles del investigador comunitario:

- El investigador activista, que se involucra directamente en un hacer irreflexivo

- El investigador especialista, cuya actividad es vista como imparcial y objetiva, desde un lugar en el que se sustenta una verdad académica que no permite incluir las voces de la comunidad

- El investigador que se convierte en pueblo, eligiendo una postura ideológica que tiene que ver con la defensa de los intereses del pueblo, con un marcado grado de mímesis con el mismo. El criterio de verdad es lo que es propio de las clases populares. Investigan para o sobre la comunidad, pero no con ella.

- El concientizador de la comunidad. Que parte de la idea de concientización como el intento de imprimir sus propios valores y modos de relacionamiento en el supuesto de que estos son los únicos válidos.

Si bien estas categorías que formula G. Perdomo están planteadas para el investigador proveniente del mundo de la academia, creemos que son útiles para reflexionar acerca de la modalidad de relación que cualquier agente que se proponga un trabajo comuni- tario establece con aquellos que son los destinatarios de sus acciones.

En ese sentido podríamos ubicar a los integrantes de la Pastoral Social en el último de los tipos descritos, el del concientizador.

Desde esta perspectiva el pedido hacia nosotros parece contener en sí mismo una contradicción: por un lado, la clara conciencia de que un vínculo asistencialista y paternalista está limitado en la posibilidad de generar cambios más o menos profundos en la calidad de vida de la gente. Pero por otro lado, fue muy elocuente el descubrimiento de que la idea que subyace es que para dejar de ser paternalistas había que cambiar a los otros en sus hábitos, su estilo de vida, sus valores, etc. sin poder comprender y tolerar la diversidad ubicándose desde un modelo cultural autoritario.

El encargo sería entonces, que con nuestras herramientas psicológicas nos hagamos cargo de "reencauzar la conducta de la gente" para que las acciones de los voluntarios pudieran tener otro eco.

Otro aspecto en el que observamos ciertos niveles de tensión y contradicción es entre enunciados que plantean un movimiento de participación e integración de los diferentes actores sociales (organizaciones formales e informales y vecinos en general) pero en los hechos cuesta delegar funciones y abrir canales de participación por no poder confiar totalmente en las capacidades de los otros.

Creemos de fundamental importancia considerar el marco institucional desde el que se desarrolla la tarea - la Iglesia - en tanto su organización estará determinando en gran medida las características de las relaciones de poder que se generan y los límites en los alcances de la intervención. Entrarían en colisión una organización de estructura jerárquica, con la pretensión de promover espacios de coordinación interinstitucional heterárquicos. 


\subsection{El sentido de la intervención}

Partimos de la base de que lo que moviliza y posibilita la intervención comunitaria es la percepción de una situación vivida como insatisfactoria, la cual oficia como motor para formular un pedido ${ }^{4}$ de ayuda. Hemos querido trabajar cómo muchos de los aspectos que marcan la relación entre los integrantes de la Capilla y los destinatarios de sus acciones, son producto de la internalización de la ideología dominante. Desde aquí, nos planteamos, como parte de la estrategia de intervención la desideologización (Montero, 1998).

Se requiere un análisis conjunto que permita investigar cuánto de la estructura social se reproduce en formas de relación no democráticas, asimétricas, con una desigual distribución del poder. Para esto se planteó problematizar las relaciones que se establecen entre los miembros de la Pastoral y los destinatarios de sus acciones. Problematizar supone "...llevar al cuestionamiento de la realidad establecida y producir conciencia..." (Montero, 1991,pág. 7)

Esta tarea se lleva adelante a través de dos caminos: por un lado señalando las contradicciones y las tensiones antes mencionadas. Trabajar las contradicciones entre el marco de referencia y las acciones, buscar analizar en el conjunto el por qué de esta contradicción, mostrar la percepción negativa que tienen de la gente con la que trabajan y la incidencia que ello tiene en la relación, han sido las líneas concretas de la intervención realizada. Por otro lado, hemos promovido la comprensión de los sectores hacia los cuales dirigen sus acciones ${ }^{5}$. El objetivo buscado es un acercamiento directo a los destinatarios para que se conviertan en sujetos de enunciación y no meramente en receptores de acciones propuestas.

En un mismo sentido problematizamos los obstáculos para integrar nuevas personas al trabajo que se realiza a pesar de la queja permanente por la sobrecarga que ello supone para unos pocos. El tema de las relaciones de poder que se establecen, los conflictos que se generan en ese marco, la dificultad para dar lugar a lo diferente y a lo nuevo, por temor a perder lo logrado, fueron algunos de los aspectos trabajados.

Posibilitar que tomen como sujetos de cambio a los miembros de la comunidad supone un proceso de concientización por parte de los voluntarios. Tomamos este concepto en un sentido distinto al de Perdomo.

Tanto el término concientización como desideologización se basan en el concepto de ideología en uno de los sentidos marxistas. Adscribimos a las definiciones que Montero formula al respecto: “...el proceso de toma de conciencia respecto de situaciones o hechos hasta ese momento ignorados"..."esa toma de conciencia supone siempre un cambio en la conciencia, que siguiendo a Goldman lleva a pasar de lo real a lo posible y a darse cuenta de que existe una situación de opresión"..."y porque conlleva un proceso de producción de conocimiento que conduce a revelar causas, a establecer conexiones, a levantar el velo de la ignorancia necesaria a un estado de cosas, acarrea entonces, un proceso de desideologización. Por tal entendemos la construcción y reconstrucción de una conciencia integral, no fraccionada, mediante la cual se produzca una comprensión del mundo en que se vive y de las circunstancias de vida en lo que tiene de totalidad". (Montero,1991.pág.9).

En la evaluación que de los talleres realiza una de las integrantes de la Pastoral Social expresa: "a mí se me vino el mundo abajo, fue un romper todo lo que estábamos haciendo para comenzar a construir algo nuevo y coherente con nuestros ideales. Un ver dónde estamos y qué tenemos..."

Se hace necesario también interrogar aquí nuestro propio lugar y la relación que establecemos con los voluntarios, así como tam- 
bién los instituidos que nos atraviesan por trabajar desde la Universidad. El lugar del saber ocupado por el docente universitario es uno de los que aparece más claramente. Nos ubicaríamos en el segundo de los tipos planteados por G. Perdomo (el investigador especialista) si nos propusiéramos disertar acerca de los aspectos centrales de la Educación Liberadora, o de las características psicosociales pertenecientes a los sectores de pobreza, o de cómo llevar adelante las transformaciones. Sería adoptar el lugar del saber y no admitir nuestra propia implicación en la generación de los cambios. Sería también asumir un aspecto del encargo de los voluntarios sin posibilitar el análisis y la reflexión.

La modalidad de relación con los voluntarios que adoptemos, se constituye en un modelo de intercambio y de construcción colectiva del conocimiento.

\subsection{Algunas consideraciones sobre el cam- bio social}

Otro de los aspectos sobre los que esta experiencia nos ha llevado a reflexionar tiene que ver con cuál es el sentido del cambio social que tanto los agentes comunitarios como nosotros pretendemos generar.

Afirmamos que trabajar hacia el cambio social es parte de la esencia de la Psicología Comunitaria entendida como una Psicología Política, siguiendo la caracterización que hace Montero (1998). De los principales lineamientos que enumera subrayamos los siguientes:

- El cambio social e individual de los agentes internos y externos como meta.

- Ese cambio debe ir acompañado de producción de conocimiento.

- El cambio se da por el proceso de "empowerment" y la creación y mantenimiento de redes de intercambio
- Se concibe a la gente como "actores sociales" que están en condiciones de decidir su destino

- La investigación como forma de intervención y la intervención como una forma de investigación con participación de la comunidad.

- Tiene un carácter político en el sentido de la producción de relaciones de poder democráticas.

Por su parte, Martín-Baró considera que el cambio social en Latinoamérica pasa por la eliminación del fatalismo, o sea: “...cambiar la relación entre la persona y su mundo, lo que supone tanto un cambio personal como un cambio social". Para esto "no sólo hace falta que modifiquen sus creencias sobre el carácter del mundo y de la vida, sino que tengan una experiencia real de modificación de su mundo y determinación de su propio futuro. Se trata de un proceso dialéctico en el que el cambio de las condiciones sociales y el cambio de las actitudes personales se posibilitan mutuamente". (MartínBaró, 1987,pág.156).

Ahora bien, nuevos procesos desde el contexto socio-histórico atraviesan a la Psicología Comunitaria, condicionando su mirada sobre el cambio y produciendo efectos a la hora de una intervención. La caída de los modelos que apuntaban a un proyecto de organización social tendiente a alcanzar mayores niveles de justicia social, los cambios en el mundo del trabajo, la generación de otras formas de marginación, la emergencia de otros actores sociales y nuevas formas de relacionamiento, son desarrolladas en "La metamorfosis de la cuestión social" por Robert Castel (1998). Analizando las características del trabajo señala que se está produciendo un ascenso de la vulnerabilidad que va de la mano con una desestabilización de los estables, ante el ingreso en una 
situación de precariedad de quienes antes habían estado integrados en el orden del trabajo.

Los trabajos comunitarios que desarrollamos quienes operamos desde la Psicología Comunitaria, son fundamentalmente con los sectores de exclusión y de vulnerabilidad. Pero lo novedoso de esta situación, y sin omitir las diferencias, es que también nosotros podemos incluirnos en esta franja de vulnerabilidad, acortando la distancia entre "nosotros" y "los otros". La búsqueda del sentido del cambio nos involucra plenamente. Esto hace que si bien hay ideales que nos sostienen, los modelos concretos están necesariamente para ser construidos con los otros más desde una postura dialógica y de investigación que desde una postura de saber que conduce a la concientización, entendida ésta como un proceso unilateral. "Nadie concientiza a nadie" parafraseando a Paulo Freire. Pero también, y en tanto que "conocer no es inventar" hay una responsabilidad social del psicólogo comunitario, ya que éste posee un saber académico que debe servir para investigar y dialogar con el saber proveniente de la vida cotidiana. Los aportes de la Educación Popular y el pensamiento gramsciano entre otros, han posibilitado la legitimación de este diálogo de saberes del que surgen nuevos conocimientos y prácticas sociales.

Paralelamente, la crisis de los paradigmas totalizantes da lugar al interjuego de lo diverso y convoca a analizar la realidad desde un pensamiento complejo, donde diversas disciplinas deben converger para dar cuenta de los fenómenos.

El imaginario social tal como lo concibe Castoriadis (1989) no es unívoco. Por un lado se nutre de posturas individualistas que plantean la salvación del más fuerte y la ausencia de la solidaridad social. Un teórico del neoliberalismo como Hayek considera como un acto de soberbia y una intromisión en "la institución perfecta del mercado" la implementación de políticas sociales compensatorias (Rebellato,1995).

Pero por otro, emergen prácticas y discursos solidarios, sostenidos por múltiples actores y movimientos sociales, que ensayan respuestas creativas a la crisis y la fragmentación.

Es sobre estos aspectos instituyentes del imaginario social que nos apoyamos en la intervención que estamos desarrollando y que nos llevó, en una segunda etapa a focalizar la tarea en el Encuentro Barrial del Buceo.

\section{Conclusiones}

Este tipo de intervención desafía a los técnicos a tener que dar cuenta de su propia comprensión del mundo y de la ciencia, comunicar su epistemología y ponerla a prueba con el modo de conocer de la comunidad con la que desarrolla su trabajo.

"Al investigador le toca ser analista del proceso comunitario en que participa, ser un pedagogo de la metodología al servicio de las necesidades comunitarias. Para ello es ineludible la exigencia fenomenológica de realizar un esfuerzo para hacer concientes al máximo sus creencias, nociones y supuestos sobre la realidad que se estudia y comunicarlas a sus interlocutores en la búsqueda de un contexto de validez intersubjetiva para el conocimiento que se aspira alcanzar..."(Perdomo,1998, pág.40).

En este sentido, también para nosotros fue una aventura poder cuestionar las matrices paternalistas de participación y abrirnos a lo que surgía, aprendiendo que hay momentos en que es bueno que "se nos caiga el mundo abajo", en la convicción de que ese es el camino hacia lo novedoso, hacia lo creativo, y en definitiva, hacia los cambios que perseguimos. 


\section{Bibliografía}

CAstel, Robert (1998). La metamorfosis de la cuestión social. Bs.As. : Paidós

CAstel, Robert (1994). De la exclusión como estado a la vulnerabilidad como proceso. Revista Archipiélago. № 21.

CAstoriadis, Cornelius. (1989). La institución imaginaria de la sociedad. En: E.Colombo. El imaginario social. Montevideo: Nordan. 27-63.

Fried Schnittman,Dora (1995). Nuevos paradigmas, cultura y subjetividad. Bs. As. : Paidós.

Martín-BARó, Ignacio (1987). El latino indolente. Carácter ideológico del fatalismo latinoamericano. En: M.Montero (coord.) Psicologia política latinoamericana. Caracas: Panapo.135-162.

Maurer Lane, Silvia y Sawaia, Bader (1991). Psicología ¿ciencia o política?. En: M. Montero (coord.) Acción y discurso. Proble- mas de Psicología Política en América Latina. Venezuela: Eduven.59-85.

Montero, Maritza (1991). Concientización, conversión y desideologización en el trabajo psicosocial comunitario. Boletín AVEPSO, XIV, 3-12.

Montero, Maritza (1998). Psychosocial community work as an alternative mode of political action (The construction and critical transformation of society). Community Work and Family. Vol 1, N 1,65-78.

Perdomo, Gloria (1998). El investigador comunitario, ¿científico imparcial o gestor del cambio social?. Boletín AVEPSO,XI, 34-41.

Rebellato, José Luis (1995). La encrucijada de la ética. Montevideo. Nordan.

RodrígueZ, Alicia y otros (1998) De ofertas y demandas. Una propuesta de intervención en Psicología Comunitaria. En IV Jornadas de Psicología Universitaria. Montevideo. Facultad de Psicología. 153-160. 